\title{
The Rahman Polynomials Are Bispectral ${ }^{\star}$
}

\author{
F. Alberto GRÜNBAUM
}

Department of Mathematics, University of California, Berkeley, CA 94720, USA

E-mail: grunbaum@math.berkeley.edu

Received February 01, 2007, in final form April 22, 2007; Published online May 03, 2007

Original article is available at http://www.emis.de/journals/SIGMA/2007/065/

\begin{abstract}
In a very recent paper, M. Rahman introduced a remarkable family of polynomials in two variables as the eigenfunctions of the transition matrix for a nontrivial Markov chain due to M. Hoare and M. Rahman. I indicate here that these polynomials are bispectral. This should be just one of the many remarkable properties enjoyed by these polynomials. For several challenges, including finding a general proof of some of the facts displayed here the reader should look at the last section of this paper.

Key words: bispectral property; multivariable polynomials; rings of commuting difference operators

2000 Mathematics Subject Classification: 33C45; 22E45
\end{abstract}

Dedicated to the memory of Vadim Kuznetsov

I first met Vadim at a meeting in Esterel, Canada back in 1994. I only saw him a few more times after that, in Amsterdam, in Leeds, in Louvain-la-Neuve, and the last time in Leganes. Although we never wrote any papers together we shared a number of common interests. I always found Vadim extremely helpful and willing to share his ideas and his vast knowledge. He was also willing to put up with people whose work was not in the mainstream and he usually would have some useful remark or a suggestion to make. I like to think that he would have enjoyed seeing this paper which builds on some remarkable work of another common friend I met for the first time at the same Esterel meeting.

\section{Preliminaries}

Starting about thirty years ago, M. Hoare and M. Rahman, published a number of papers studying a class of statistical models that were nicely associated with "classical" orthogonal polynomials in one variable. The reader can consult $[19,20,21,4]$.

In a very recent paper [22], which I have seen before publication thanks to the kindness of M. Rahman, they take a huge step and consider the case of several variables. I propose to call these statistical models with the name Hoare-Rahman, and to call the multivariable polynomials that arise with the name Rahman polynomials. I am thankful to Prof. Rahman for several inspiring conversations on the contents of [22], in different coffee houses and restaurants in Fremont, California on the last days of 2006.

The model of interest here captures very well the idea of a game of chance where the player takes a risk that can never improve his/her winnings but is then given a second opportunity to improve his/her lot. I am convinced that models of this kind should be of great interest in several areas of applied mathematics not only in physics (where they were conceived) but also in biology, population dynamics, evolutionary models, etc. On top of their potential for applications the polynomials discovered by M. Rahman are a piece of beauty. I hope that this

\footnotetext{
^This paper is a contribution to the Vadim Kuznetsov Memorial Issue 'Integrable Systems and Related Topics'.
} The full collection is available at http://www.emis.de/journals/SIGMA/kuznetsov.html 
paper will incite other people to look around for the gems contained in [22]. I only recall the points from this paper that are needed in the present context, urging the reader to go back to the source for inspiration.

For a systematic study of polynomials in several variables one should consult [8] as well as the work on Macdonald polynomials of various kinds, see [31]. A look at the pioneering work of Tom Koornwinder, see for instance [28], is always a very good idea.

\section{The Rahman polynomials}

Given generic values of the parameters $p_{1}, p_{2}, p_{3}, p_{4}$ (these could even be complex numbers) define $t, u, v, w$ by means of

$$
\begin{aligned}
t & =\frac{\left(p_{1}+p_{2}\right)\left(p_{1}+p_{3}\right)}{p_{1}\left(p_{1}+p_{2}+p_{3}+p_{4}\right)}, & u & =\frac{\left(p_{1}+p_{3}\right)\left(p_{4}+p_{3}\right)}{p_{3}\left(p_{1}+p_{2}+p_{3}+p_{4}\right)}, \\
v & =\frac{\left(p_{1}+p_{2}\right)\left(p_{2}+p_{4}\right)}{p_{2}\left(p_{1}+p_{2}+p_{3}+p_{4}\right)}, & w & =\frac{\left(p_{4}+p_{2}\right)\left(p_{4}+p_{3}\right)}{p_{4}\left(p_{1}+p_{2}+p_{3}+p_{4}\right)} .
\end{aligned}
$$

Now we can define with M. Rahman the polynomials in $x, y$ given by

$$
P_{m, n}(x, y)=\sum_{i} \sum_{j} \sum_{k} \sum_{\ell} \frac{(-m)_{i+j}(-n)_{k+\ell}(-x)_{i+k}(-y)_{j+\ell}}{i ! j ! k ! \ell !(-N)_{i+j+k+\ell}} t^{i} u^{j} v^{k} w^{\ell} .
$$

I will not try to explain M. Rahman's path to these polynomials, but the reader should consult [22]. In particular one finds in [22] a discussion of the connection with the $9-j$ symbols used in quantum mechanics.

\section{The Hoare-Rahman model and the kernel $\mathcal{K}$}

Denote by

$$
b(k, N, \alpha)=\left(\begin{array}{c}
N \\
k
\end{array}\right) \alpha^{k}(1-\alpha)^{N-k}
$$

the usual binomial distribution giving the probability of $k$ successes in $N$ repetitions of an experiment with two possible outcomes: success (with probability $\alpha$ ) and failure (with probability $1-\alpha)$. Likewise denote by $b_{2}\left(i_{1}, i_{2}, N ; \beta_{1}, \beta_{2}\right)$ the trinomial distribution with parameters $\beta_{1}, \beta_{2}$.

This means that that for $i_{1}, i_{2}$ nonnegative and such that $i_{1}+i_{2} \leq N$ we have

$$
b_{2}\left(i_{1}, i_{2}, N ; \beta_{1}, \beta_{2}\right)=\beta_{1}^{i_{1}} \beta_{2}^{i_{2}}\left(1-\beta_{1}-\beta_{2}\right)^{N-i_{1}-i_{2}}\left(\frac{N !}{i_{1} ! i_{2} !\left(N-i_{1}-i_{2}\right) !}\right) .
$$

Define now a transition probability kernel

$$
\mathcal{K}\left(j_{1}, j_{2} ; i_{1}, i_{2}\right)
$$

in the space of non-negative integer physical variables $i_{1}, i_{2}$ and $j_{1}, j_{2}$ such that

$$
i_{1}+i_{2} \leq N, \quad j_{1}+j_{2} \leq N
$$

by means of the expression

$$
\mathcal{K}\left(j_{1}, j_{2} ; i_{1}, i_{2}\right)=\sum_{k_{1}=0}^{\min \left(i_{1}, j_{1}\right)} \sum_{k_{2}=0}^{\min \left(i_{2}, j_{2}\right)} b\left(k_{1}, i_{1} ; \alpha_{1}\right) b\left(k_{2}, i_{2} ; \alpha_{2}\right)
$$




$$
\times b_{2}\left(j_{1}-k_{1}, j_{2}-k_{2}, N-k_{1}-k_{2} ; \beta_{1}, \beta_{2}\right) .
$$

The expression above is written so as to agree with the one in [22]. Notice that its interpretation is that of the probability of a transition from the state $\left(i_{1}, i_{2}\right)$ to the new sate $\left(j_{1}, j_{2}\right)$ in one step (consisting of three tosses) of our process, as will be explained in the next section.

At this point the parameters $\alpha_{1}, \alpha_{2}, \beta_{1}, \beta_{2}$ are free to lie anywhere in the interval $[0,1]$, with the sum of $\beta_{1}$ and $\beta_{2}$ not exceeding the value 1 .

The corresponding Markov chain is not a "nearest neighbour one" in the sense that the classical Ehrenfest urn model, see [9, 10, 25, 32], or all other classical models are. Here the transition probability matrix has all its entries positive and therefore there is no sense in which a one-step transition moves you only to your immediate neighbours. I find it remarkable that the complete spectral problem can be solved for such a kernel, but this is exactly what the Rahman polynomials do, at least under certain extra conditions.

\section{Poker dice}

A concrete example of the model is provided by a gambling game known as Poker dice. The version considered by M. Hoare and M. Rahman goes as follows. You have $N$ identical dice with $n$ faces each. There are three kinds of faces: red, black and "blanks". The probabilities of getting a red or a black face when rolling one dice are $\alpha_{1}$ and $\alpha_{2}$, respectively. The state of the system is given by $\left(i_{1}, i_{2}\right)$, the number of red and black faces among the $N$ dice. Think of the remaining $N-i_{1}-i_{2}$ dice as failures.

Start form the state $\left(i_{1}, i_{2}\right)$ and get ready to roll three times. The resulting number of reds and blacks after these three rools will be denoted by $\left(j_{1}, j_{2}\right)$. This will constitute one time step in the Markov chain to be described now.

The $i_{1}$ dice that show red are tossed and yield $k_{1}$ red faces. The $i_{2}$ dice that showed a black face are tossed and yield $k_{2}$ blacks. Now you take the initial $N-i_{1}-i_{2}$ blanks and combine them with the $i_{1}-k_{1}$ dice that failed to reproduce their original red color and the $i_{2}-k_{2}$ dice that failed to reproduce their black color. The total number of "failures" is now $N-k_{1}-k_{2}$. These dice are thrown by themselves: they yield a certain number of red and black faces which are added to the $k_{1}$ red faced dice and $k_{2}$ black faced dice that were saved earlier. This total number of reds is now $j_{1}$ and the total number of blacks is $j_{2}$. To obtain full generality the probabilities of getting a red or a black for each individual dice on this last toss can be taken to be $\beta_{1}$ and $\beta_{2}$, not necessarily the old values $\alpha_{1}$ and $\alpha_{2}$.

The case of $N=5$ is referred to as standard "poker dice" in [22] and will be used to illustrate later on the main point in this paper, namely the bispectral property of the Rahman polynomials. We have verified this property for many other values of $N$, but we do not have a nice general expression for the operator in question, or a nice proof of its existence for general $N$.

\section{The orthogonality weight}

In [22] one sees that the Rahman polynomials are orthogonal with respect to the trinomial distribution

$$
b_{2}\left(x, y ; N, \eta_{1}, \eta_{2}\right) \text {, }
$$

where the parameters $\eta_{1}, \eta_{2}$ are given in terms of $p_{1}, p_{2}, p_{3}, p_{4}$ by means of

$$
\eta_{1}=\frac{p_{1} p_{2}\left(p_{1}+p_{2}+p_{3}+p_{4}\right)}{\left(p_{1}+p_{2}\right)\left(p_{1}+p_{3}\right)\left(p_{2}+p_{4}\right)}, \quad \eta_{2}=\frac{p_{3} p_{4}\left(p_{1}+p_{2}+p_{3}+p_{4}\right)}{\left(p_{1}+p_{3}\right)\left(p_{4}+p_{2}\right)\left(p_{4}+p_{3}\right)} .
$$


Given this weight, for an arbitrary choice of the parameters $\eta_{1}, \eta_{2}$, one could blindly decide to "order" the set of monomials

$$
x^{i} y^{j}
$$

and then apply the Gram-Schmidt process to obtain orthogonal polynomials. Of course there is no unique way to order these monomials and each ordering will produce a different family of polynomials.

One of the features of the work of M. Rahman is that it automatically gives one set of polynomials that are already orthogonal and, as we see in [22] and here, enjoy remarkable properties. Moreover he produces a four parameter family of these polynomials, having started from a the two parameters $\eta_{1}, \eta_{2}$. There is clearly a lot of inspiration behind this construction, and the reader should consult [22].

\section{The bispectral property}

Recall that $\mathcal{K}$ depends on one set of four parameters and the Rahman polynomials on a different set of four parameters.

As shown in [22], the Rahman polynomials satisfy the eigenvalue relation

$$
\mathcal{K} P_{m, n}(x, y)=\lambda_{m, n} P_{m, n}(x, y)
$$

for $(x, y)$ in "physical space", i.e. $x, y$ are integers satisfying $x \geq 0, y \geq 0, x+y \leq N$, with

$$
\begin{aligned}
\lambda_{m, n}= & \left\{\frac{1-\alpha_{1} \eta_{1}-\alpha_{2} \eta_{2}-\eta_{1} t\left(1-\alpha_{1}\right)-\eta_{2} u\left(1-\alpha_{2}\right)}{1-\alpha_{1} \eta_{1}-\alpha_{2} \eta_{2}}\right\}^{m} \\
& \times\left\{\frac{1-\alpha_{1} \eta_{1}-\alpha_{2} \eta_{2}-\eta_{1} v\left(1-\alpha_{1}\right)-\eta_{2} w\left(1-\alpha_{2}\right)}{1-\alpha_{1} \eta_{1}-\alpha_{2} \eta_{2}}\right\}^{n} \\
= & \left(1-\beta_{1} t-\beta_{2} u\right)^{m}\left(1-\beta_{1} v-\beta_{2} w\right)^{n} .
\end{aligned}
$$

More explicitly, with the definitions made above this reads,

$$
\sum_{j_{1}, j_{2}} K\left(j_{1}, j_{2} ; i_{1}, i_{2}\right) P_{m, n}\left(j_{1}, j_{2}\right)=\lambda_{m, n} P_{m, n}\left(i_{1}, i_{2}\right)
$$

For this to be true we need to impose some relations connecting the parameters $p_{1}, p_{2}, p_{3}, p_{4}$ and $\alpha_{1}, \alpha_{2}, \beta_{1}, \beta_{2}$. This issue has been addressed in [22].

In the considerations above we are thinking of these polynomials as functions of the physical space variables $x, y$ for fixed values of the frequency parameters $m, n$.

In the spirit of [5] one could ask if they are the eigenvectors of another spectral problem when considered, for fixed $x, y$, as functions of $m, n$. If this were true we would have an analog of the three-term recursion relation satisfied by orthogonal polynomials of one variable. In the continuous-continuous case this question was raised in [5] for a second order Schrödinger type differential operator. For many ramifications of this problem the reader can see [18].

We give below the simplest recursion that we have been able to find. To be very concrete we display the result in the case of $N=5$ which corresponds to the Poker dice example described above. The operator in question consists of multiplication by the function $\left(p_{1}+p_{2}\right) x-\left(p_{3}+p_{4}\right) y$ and it gives a "five-point" formula, where most points in the "spectral space" have four neighbours. There are, as indicated above, similar recursions for other values of $N$ but we do not have a general expression that would cover all cases.

In the case of $N=5$ the frequency space (and also the physical space) looks as follows 
If we declare two points to be adjacent when they can be obtained by going one step either in the vertical or horizontal directions, and we order the 21 states as follows

$$
\begin{aligned}
& (0,0),(1,0),(2,0),(3,0),(4,0),(5,0),(0,1),(1,1),(2,1),(3,1),(4,1), \\
& (0,2),(1,2),(2,2),(3,2),(0,3),(1,3),(2,3),(0,4),(1,4),(0,5)
\end{aligned}
$$

then one can work out easily the adjacency matrix.

The matrix $\mathcal{B}$ to be detailed below will have nonzero entries exactly where the adjacency matrix has a 1. It is therefore as much of a local matrix as one can get in this setup. It will correspond to the analog of the three term recursion relation of the usual situation when one deals with orthogonal polynomials in one variable.

We claim that the Rahman polynomials, as functions of $m, n$ satisfy the difference equation

$$
\mathcal{B} P_{m, n}(x, y)=\left(\left(p_{1}+p_{2}\right) x-\left(p_{3}+p_{4}\right) y\right) P_{m, n}(x, y)
$$

as long as $(x, y)$ lie in physical space.

Here the matrix $\mathcal{B}$ is made up of rows as specified one at a time below. Notice that in each row there is an element denoted by $s_{i}$ for which we do not give an expression. This is not needed since the sum of all the entries in each row equals the value zero.

$$
\left[s_{1}, a_{1}, 0,0,0,0, b_{1}, 0,0,0,0,0,0,0,0,0,0,0,0,0,0\right]
$$

with

$$
\begin{aligned}
& a_{1}=\frac{5 p_{1} p_{3}\left(p_{4}+p_{2}\right)\left(p_{4}+p_{3}+p_{2}+p_{1}\right)}{\left(p_{3}+p_{1}\right)\left(p_{1} p_{4}-p_{2} p_{3}\right)}, \quad b_{1}=-\frac{5 p_{2}\left(p_{3}+p_{1}\right) p_{4}\left(p_{4}+p_{3}+p_{2}+p_{1}\right)}{\left(p_{4}+p_{2}\right)\left(p_{1} p_{4}-p_{2} p_{3}\right)} \\
& {\left[a_{2}, s_{2}, c_{2}, 0,0,0,0, d_{2}, 0,0,0,0,0,0,0,0,0,0,0,0,0\right]}
\end{aligned}
$$

with

$$
\begin{aligned}
& a_{2}=\frac{p_{1} p_{4}-p_{2} p_{3}}{p_{3}+p_{1}}, \quad c_{2}=\frac{4 p_{1} p_{3}\left(p_{4}+p_{2}\right)\left(p_{4}+p_{3}+p_{2}+p_{1}\right)}{\left(p_{3}+p_{1}\right)\left(p_{1} p_{4}-p_{2} p_{3}\right)}, \\
& d_{2}=-\frac{4 p_{2}\left(p_{3}+p_{1}\right) p_{4}\left(p_{4}+p_{3}+p_{2}+p_{1}\right)}{\left(p_{4}+p_{2}\right)\left(p_{1} p_{4}-p_{2} p_{3}\right)} \\
& {\left[0, a_{3}, s_{3}, c_{3}, 0,0,0,0, d_{3}, 0,0,0,0,0,0,0,0,0,0,0,0\right]}
\end{aligned}
$$

with

$$
a_{3}=\frac{2\left(p_{1} p_{4}-p_{2} p_{3}\right)}{p_{3}+p_{1}}, \quad c_{3}=\frac{3 p_{1} p_{3}\left(p_{4}+p_{2}\right)\left(p_{4}+p_{3}+p_{2}+p_{1}\right)}{\left(p_{3}+p_{1}\right)\left(p_{1} p_{4}-p_{2} p_{3}\right)}
$$




$$
\begin{aligned}
& d_{3}=-\frac{3 p_{2}\left(p_{3}+p_{1}\right) p_{4}\left(p_{4}+p_{3}+p_{2}+p_{1}\right)}{\left(p_{4}+p_{2}\right)\left(p_{1} p_{4}-p_{2} p_{3}\right)} \\
& {\left[0,0, a_{4}, s_{4}, c_{4}, 0,0,0,0, d_{4}, 0,0,0,0,0,0,0,0,0,0,0\right]}
\end{aligned}
$$

with

$$
\begin{aligned}
& a_{4}=\frac{3\left(p_{1} p_{4}-p_{2} p_{3}\right)}{p_{3}+p_{1}}, \quad c_{4}=\frac{2 p_{1} p_{3}\left(p_{4}+p_{2}\right)\left(p_{4}+p_{3}+p_{2}+p_{1}\right)}{\left(p_{3}+p_{1}\right)\left(p_{1} p_{4}-p_{2} p_{3}\right)}, \\
& d_{4}=-\frac{2 p_{2}\left(p_{3}+p_{1}\right) p_{4}\left(p_{4}+p_{3}+p_{2}+p_{1}\right)}{\left(p_{4}+p_{2}\right)\left(p_{1} p_{4}-p_{2} p_{3}\right)} \\
& {\left[0,0,0, a_{5}, s_{5}, c_{5}, 0,0,0,0, e_{5}, 0,0,0,0,0,0,0,0,0,0\right]}
\end{aligned}
$$

with

$$
\begin{aligned}
& a_{5}=\frac{4\left(p_{1} p_{4}-p_{2} p_{3}\right)}{p_{3}+p_{1}}, \quad c_{5}=\frac{p_{1} p_{3}\left(p_{4}+p_{2}\right)\left(p_{4}+p_{3}+p_{2}+p_{1}\right)}{\left(p_{3}+p_{1}\right)\left(p_{1} p_{4}-p_{2} p_{3}\right)}, \\
& e_{5}=-\frac{p_{2}\left(p_{3}+p_{1}\right) p_{4}\left(p_{4}+p_{3}+p_{2}+p_{1}\right)}{\left(p_{4}+p_{2}\right)\left(p_{1} p_{4}-p_{2} p_{3}\right)} \\
& {\left[0,0,0,0, a_{6}, s_{6}, 0,0,0,0,0,0,0,0,0,0,0,0,0,0,0\right]}
\end{aligned}
$$

with

$$
\begin{aligned}
& a_{6}=\frac{5\left(p_{1} p_{4}-p_{2} p_{3}\right)}{p_{3}+p_{1}} ; \\
& {\left[d_{7}, 0,0,0,0,0, s_{7}, c_{7}, 0,0,0, a_{7}, 0,0,0,0,0,0,0,0,0\right]}
\end{aligned}
$$

with

$$
\begin{aligned}
& a_{7}=-\frac{4 p_{2}\left(p_{3}+p_{1}\right) p_{4}\left(p_{4}+p_{3}+p_{2}+p_{1}\right)}{\left(p_{4}+p_{2}\right)\left(p_{1} p_{4}-p_{2} p_{3}\right)}, \\
& c_{7}=\frac{4 p_{1} p_{3}\left(p_{4}+p_{2}\right)\left(p_{4}+p_{3}+p_{2}+p_{1}\right)}{\left(p_{3}+p_{1}\right)\left(p_{1} p_{4}-p_{2} p_{3}\right)}, \quad d_{7}=-\frac{p_{1} p_{4}-p_{2} p_{3}}{p_{4}+p_{2}} ; \\
& {\left[0, a_{8}, 0,0,0,0, b_{8}, s_{8}, d_{8}, 0,0,0, e_{8}, 0,0,0,0,0,0,0,0\right]}
\end{aligned}
$$

with

$$
\begin{aligned}
& a_{8}=-\frac{p_{1} p_{4}-p_{2} p_{3}}{p_{4}+p_{2}}, \quad b_{8}=\frac{p_{1} p_{4}-p_{2} p_{3}}{p_{3}+p_{1}}, \\
& d_{8}=\frac{3 p_{1} p_{3}\left(p_{4}+p_{2}\right)\left(p_{4}+p_{3}+p_{2}+p_{1}\right)}{\left(p_{3}+p_{1}\right)\left(p_{1} p_{4}-p_{2} p_{3}\right)}, \quad e_{8}=-\frac{3 p_{2}\left(p_{3}+p_{1}\right) p_{4}\left(p_{4}+p_{3}+p_{2}+p_{1}\right)}{\left(p_{4}+p_{2}\right)\left(p_{1} p_{4}-p_{2} p_{3}\right)} ; \\
& {\left[0,0, e_{9}, 0,0,0,0, c_{9}, s_{9}, a_{9}, 0,0,0, d_{9}, 0,0,0,0,0,0,0\right]}
\end{aligned}
$$

with

$$
\begin{aligned}
& a_{9}=\frac{2 p_{1} p_{3}\left(p_{4}+p_{2}\right)\left(p_{4}+p_{3}+p_{2}+p_{1}\right)}{\left(p_{3}+p_{1}\right)\left(p_{1} p_{4}-p_{2} p_{3}\right)}, \quad c_{9}=\frac{2\left(p_{1} p_{4}-p_{2} p_{3}\right)}{p_{3}+p_{1}}, \\
& d_{9}=-\frac{2 p_{2}\left(p_{3}+p_{1}\right) p_{4}\left(p_{4}+p_{3}+p_{2}+p_{1}\right)}{\left(p_{4}+p_{2}\right)\left(p_{1} p_{4}-p_{2} p_{3}\right)}, \quad e_{9}=-\frac{p_{1} p_{4}-p_{2} p_{3}}{p_{4}+p_{2}} ; \\
& {\left[0,0,0, d_{10}, 0,0,0,0, a_{10}, s_{10}, c_{10}, 0,0,0, e_{10}, 0,0,0,0,0,0\right]}
\end{aligned}
$$

with

$$
a_{10}=\frac{3\left(p_{1} p_{4}-p_{2} p_{3}\right)}{p_{3}+p_{1}}, \quad c_{10}=\frac{p_{1} p_{3}\left(p_{4}+p_{2}\right)\left(p_{4}+p_{3}+p_{2}+p_{1}\right)}{\left(p_{3}+p_{1}\right)\left(p_{1} p_{4}-p_{2} p_{3}\right)},
$$




$$
\begin{aligned}
& d_{10}=-\frac{p_{1} p_{4}-p_{2} p_{3}}{p_{4}+p_{2}}, \quad e_{10}=-\frac{p_{2}\left(p_{3}+p_{1}\right) p_{4}\left(p_{4}+p_{3}+p_{2}+p_{1}\right)}{\left(p_{4}+p_{2}\right)\left(p_{1} p_{4}-p_{2} p_{3}\right)} \\
& {\left[0,0,0,0, b_{11}, 0,0,0,0, a_{11}, s_{11}, 0,0,0,0,0,0,0,0,0,0\right]}
\end{aligned}
$$

with

$$
\begin{aligned}
& a_{11}=\frac{4\left(p_{1} p_{4}-p_{2} p_{3}\right)}{p_{3}+p_{1}}, \quad b_{11}=-\frac{p_{1} p_{4}-p_{2} p_{3}}{p_{4}+p_{2}} \\
& {\left[0,0,0,0,0,0, a_{12}, 0,0,0,0, s_{12}, d_{12}, 0,0, c_{12}, 0,0,0,0,0\right]}
\end{aligned}
$$

with

$$
\begin{aligned}
& a_{12}=-\frac{2\left(p_{1} p_{4}-p_{2} p_{3}\right)}{p_{4}+p_{2}}, \quad c_{12}=-\frac{3 p_{2}\left(p_{3}+p_{1}\right) p_{4}\left(p_{4}+p_{3}+p_{2}+p_{1}\right)}{\left(p_{4}+p_{2}\right)\left(p_{1} p_{4}-p_{2} p_{3}\right)}, \\
& d_{12}=\frac{3 p_{1} p_{3}\left(p_{4}+p_{2}\right)\left(p_{4}+p_{3}+p_{2}+p_{1}\right)}{\left(p_{3}+p_{1}\right)\left(p_{1} p_{4}-p_{2} p_{3}\right)} \\
& {\left[0,0,0,0,0,0,0, c_{13}, 0,0,0, a_{13}, s_{13}, d_{13}, 0,0, e_{13}, 0,0,0,0\right]}
\end{aligned}
$$

with

$$
\begin{aligned}
& a_{13}=\frac{p_{1} p_{4}-p_{2} p_{3}}{p_{3}+p_{1}}, \quad c_{13}=-\frac{2\left(p_{1} p_{4}-p_{2} p_{3}\right)}{p_{4}+p_{2}}, \\
& d_{13}=\frac{2 p_{1} p_{3}\left(p_{4}+p_{2}\right)\left(p_{4}+p_{3}+p_{2}+p_{1}\right)}{\left(p_{3}+p_{1}\right)\left(p_{1} p_{4}-p_{2} p_{3}\right)}, \quad e_{13}=-\frac{2 p_{2}\left(p_{3}+p_{1}\right) p_{4}\left(p_{4}+p_{3}+p_{2}+p_{1}\right)}{\left(p_{4}+p_{2}\right)\left(p_{1} p_{4}-p_{2} p_{3}\right)} ; \\
& {\left[0,0,0,0,0,0,0,0, d_{14}, 0,0,0, a_{14}, s_{14}, c_{14}, 0,0, e_{14}, 0,0,0\right]}
\end{aligned}
$$

with

$$
\begin{aligned}
& a_{14}=\frac{2\left(p_{1} p_{4}-p_{2} p_{3}\right)}{p_{3}+p_{1}}, \quad c_{14}=\frac{p_{1} p_{3}\left(p_{4}+p_{2}\right)\left(p_{4}+p_{3}+p_{2}+p_{1}\right)}{\left(p_{3}+p_{1}\right)\left(p_{1} p_{4}-p_{2} p_{3}\right)}, \\
& d_{14}=-\frac{2\left(p_{1} p_{4}-p_{2} p_{3}\right)}{p_{4}+p_{2}}, \\
& {\left[0,0,0,0,0,0,0,0,0, a_{15}, 0,0,0, c_{15}, s_{15}, 0,0,0,0,0,0\right]}
\end{aligned}
$$

with

$$
\begin{aligned}
& a_{15}=-\frac{2\left(p_{1} p_{4}-p_{2} p_{3}\right)}{p_{4}+p_{2}}, \quad c_{15}=\frac{3\left(p_{1} p_{4}-p_{2} p_{3}\right)}{p_{3}+p_{1}} \\
& {\left[0,0,0,0,0,0,0,0,0,0,0, a_{16}, 0,0,0, s_{16}, c_{16}, 0, d_{16}, 0,0\right]}
\end{aligned}
$$

with

$$
\begin{aligned}
& a_{16}=-\frac{3\left(p_{1} p_{4}-p_{2} p_{3}\right)}{p_{4}+p_{2}}, \quad c_{16}=\frac{2 p_{1} p_{3}\left(p_{4}+p_{2}\right)\left(p_{4}+p_{3}+p_{2}+p_{1}\right)}{\left(p_{3}+p_{1}\right)\left(p_{1} p_{4}-p_{2} p_{3}\right)}, \\
& d_{16}=-\frac{2 p_{2}\left(p_{3}+p_{1}\right) p_{4}\left(p_{4}+p_{3}+p_{2}+p_{1}\right)}{\left(p_{4}+p_{2}\right)\left(p_{1} p_{4}-p_{2} p_{3}\right)} ; \\
& {\left[0,0,0,0,0,0,0,0,0,0,0,0, e_{17}, 0,0, a_{17}, s_{17}, c_{17}, 0, d_{17}, 0\right]}
\end{aligned}
$$

with

$$
\begin{aligned}
& a_{17}=\frac{p_{1} p_{4}-p_{2} p_{3}}{p_{3}+p_{1}}, \quad c_{17}=\frac{p_{1} p_{3}\left(p_{4}+p_{2}\right)\left(p_{4}+p_{3}+p_{2}+p_{1}\right)}{\left(p_{3}+p_{1}\right)\left(p_{1} p_{4}-p_{2} p_{3}\right)} \\
& d_{17}=-\frac{p_{2}\left(p_{3}+p_{1}\right) p_{4}\left(p_{4}+p_{3}+p_{2}+p_{1}\right)}{\left(p_{4}+p_{2}\right)\left(p_{1} p_{4}-p_{2} p_{3}\right)}, \quad e_{17}=-\frac{3\left(p_{1} p_{4}-p_{2} p_{3}\right)}{p_{4}+p_{2}}
\end{aligned}
$$




$$
\left[0,0,0,0,0,0,0,0,0,0,0,0,0, a_{18}, 0,0, c_{18}, s_{18}, 0,0,0\right]
$$

with

$$
\begin{aligned}
& a_{18}=-\frac{3\left(p_{1} p_{4}-p_{2} p_{3}\right)}{p_{4}+p_{2}}, \quad c_{18}=\frac{2\left(p_{1} p_{4}-p_{2} p_{3}\right)}{p_{3}+p_{1}} \\
& {\left[0,0,0,0,0,0,0,0,0,0,0,0,0,0,0, c_{19}, 0,0, s_{19}, a_{19}, d_{19}\right]}
\end{aligned}
$$

with

$$
\begin{aligned}
& a_{19}=\frac{p_{1} p_{3}\left(p_{4}+p_{2}\right)\left(p_{4}+p_{3}+p_{2}+p_{1}\right)}{\left(p_{3}+p_{1}\right)\left(p_{1} p_{4}-p_{2} p_{3}\right)} \\
& c_{19}=-\frac{4\left(p_{1} p_{4}-p_{2} p_{3}\right)}{p_{4}+p_{2}}, \quad d_{19}=-\frac{p_{2}\left(p_{3}+p_{1}\right) p_{4}\left(p_{4}+p_{3}+p_{2}+p_{1}\right)}{\left(p_{4}+p_{2}\right)\left(p_{1} p_{4}-p_{2} p_{3}\right)} ; \\
& {\left[0,0,0,0,0,0,0,0,0,0,0,0,0,0,0,0, a_{20}, 0, c_{20}, s_{20}, 0\right]}
\end{aligned}
$$

with

$$
\begin{aligned}
& a_{20}=-\frac{4\left(p_{1} p_{4}-p_{2} p_{3}\right)}{p_{4}+p_{2}}, \quad c_{20}=\frac{p_{1} p_{4}-p_{2} p_{3}}{p_{3}+p_{1}} \\
& {\left[0,0,0,0,0,0,0,0,0,0,0,0,0,0,0,0,0,0, a_{21}, 0, s_{21}\right]}
\end{aligned}
$$

with

$$
a_{21}=-\frac{5\left(p_{1} p_{4}-p_{2} p_{3}\right)}{p_{4}+p_{2}} .
$$

We note that these relations hold for arbitrary (generic) values of $p_{1}, p_{2}, p_{3}, p_{4}$.

If one considers multiplication by $x$ or by $y$ we get a "seven-point" formula. Of course it would be interesting, in the spirit of [3] to consider the full algebra of difference operators going along with the Rahman polynomials.

Thinking along these lines one could also consider the full algebra of difference operators that go along with the Rahman polynomials when thought of as functions of $x, y$. This simply means the algebra of all matrices that commute with the kernel $\mathcal{K}$. While the original kernel $\mathcal{K}$ has a very nice probabilistic interpretation there is no reason why other operators in this algebra should allow such an interpretation. More on this in the next section.

\section{Global vs. local}

One should naturally think of the kernel $\mathcal{K}$ given earlier as a global operator, one that in this discrete case is represented by a full matrix and that in the continuous case would be represented by an integral operator. At the core of many considerations leading up to the bispectral problem is the issue of the existence of a local operator that would commute with the global one. This remarkable piece of work is forever associated with the names of D. Slepian, H. Landau and H. Pollack. This is discussed for instance in [13, 14].

Here, as an illustration we show that this question has a positive answer in the setup of Hoare and Rahman. Take $N=3$ in which case we can see, for arbitrary choice of the free parameters $\alpha_{1}, \alpha_{2}, \beta_{1}, \beta_{2}$ that the matrix given below is essentially the only one that commutes with the kernel $\mathcal{K}$. Once again, we have checked that this holds for other values of $N$, and this case is used here only for illustration purposes.

In the spirit of the work just alluded to one should consider $\mathcal{K}$ as the analog of the operator of time-band-time limiting and the operator below as the analog of the second order differential 
operator that comes from separation of variables for the Laplacian in $\mathbb{R}^{3}$ in prolate spheroidal coordinates.

In agreement with the case $N=5$ considered as an illustration earlier, we order the states in physical space as follows

$$
(0,0),(1,0),(2,0),(3,0),(0,1),(1,1),(2,1),(0,2),(1,2),(0,3) \text {. }
$$

Then, up to trivial scaling and shifts the only local matrix that commutes with $\mathcal{K}$ is given by

$$
\left[\begin{array}{cccccccccc}
x_{1} & x_{2} & 0 & 0 & x_{3} & 0 & 0 & 0 & 0 & 0 \\
x_{4} & x_{5} & x_{6} & 0 & 0 & x_{7} & 0 & 0 & 0 & 0 \\
0 & x_{8} & x_{9} & x_{10} & 0 & 0 & x_{11} & 0 & 0 & 0 \\
0 & 0 & x_{12} & x_{13} & 0 & 0 & 0 & 0 & 0 & 0 \\
x_{14} & 0 & 0 & 0 & x_{15} & x_{16} & 0 & x_{17} & 0 & 0 \\
0 & x_{18} & 0 & 0 & x_{19} & x_{20} & x_{21} & 0 & x_{22} & 0 \\
0 & 0 & x_{23} & 0 & 0 & x_{24} & x_{25} & 0 & 0 & 0 \\
0 & 0 & 0 & 0 & x_{26} & 0 & 0 & x_{27} & x_{28} & x_{29} \\
0 & 0 & 0 & 0 & 0 & x_{30} & 0 & x_{31} & x_{32} & 0 \\
0 & 0 & 0 & 0 & 0 & 0 & 0 & x_{33} & 0 & x_{34}
\end{array}\right]
$$

as long as we choose its entries as follows

$$
\begin{aligned}
& x_{1}=3\left(\alpha_{1} \alpha_{2} \beta_{2}-2 \alpha_{1} \beta_{2}+\alpha_{1} \alpha_{2} \beta_{1}-\alpha_{2} \beta_{1}-\alpha_{1} \beta_{1}-\alpha_{1} \alpha_{2}+\alpha_{1}\right) /\left(\alpha_{1} \beta_{2}\right) \text {, } \\
& x_{2}=\frac{3 \alpha_{2} \beta_{1}}{\alpha_{1} \beta_{2}} \quad x_{3}=3, \quad x_{4}=\frac{\left(\alpha_{1}-1\right) \alpha_{2}\left(\beta_{2}+\beta_{1}-1\right)}{\alpha_{1} \beta_{2}}, \\
& x_{5}=\left(2 \alpha_{1} \alpha_{2} \beta_{2}+\alpha_{2} \beta_{2}-5 \alpha_{1} \beta_{2}+2 \alpha_{1} \alpha_{2} \beta_{1}-\alpha_{2} \beta_{1}-3 \alpha_{1} \beta_{1}-2 \alpha_{1} \alpha_{2}-\alpha_{2}+3 \alpha_{1}\right) /\left(\alpha_{1} \beta_{2}\right) \text {, } \\
& x_{6}=\frac{2 \alpha_{2} \beta_{1}}{\alpha_{1} \beta_{2}}, \quad x_{7}=2, \quad x_{8}=\frac{2\left(\alpha_{1}-1\right) \alpha_{2}\left(\beta_{2}+\beta_{1}-1\right)}{\alpha_{1} \beta_{2}}, \\
& x_{9}=\left(\alpha_{1} \alpha_{2} \beta_{2}+2 \alpha_{2} \beta_{2}-4 \alpha_{1} \beta_{2}+\alpha_{1} \alpha_{2} \beta_{1}+\alpha_{2} \beta_{1}-3 \alpha_{1} \beta_{1}-\alpha_{1} \alpha_{2}-2 \alpha_{2}+3 \alpha_{1}\right) /\left(\alpha_{1} \beta_{2}\right) \text {, } \\
& x_{10}=\frac{\alpha_{2} \beta_{1}}{\alpha_{1} \beta_{2}}, \quad x_{11}=1, \quad x_{12}=\frac{3\left(\alpha_{1}-1\right) \alpha_{2}\left(\beta_{2}+\beta_{1}-1\right)}{\alpha_{1} \beta_{2}} \text {, } \\
& x_{13}=\frac{3\left(\alpha_{2}-\alpha_{1}\right)\left(\beta_{2}+\beta_{1}-1\right)}{\alpha_{1} \beta_{2}}, \quad x_{14}=\frac{\left(\alpha_{2}-1\right)\left(\beta_{2}+\beta_{1}-1\right)}{\beta_{2}}, \\
& x_{15}=2\left(\alpha_{1} \alpha_{2} \beta_{2}-2 \alpha_{1} \beta_{2}+\alpha_{1} \alpha_{2} \beta_{1}-\alpha_{2} \beta_{1}-\alpha_{1} \beta_{1}-\alpha_{1} \alpha_{2}+\alpha_{1}\right) /\left(\alpha_{1} \beta_{2}\right) \text {, } \\
& x_{16}=\frac{2 \alpha_{2} \beta_{1}}{\alpha_{1} \beta_{2}}, \quad x_{17}=2, \quad x_{18}=\frac{\left(a_{2}-1\right)\left(\beta_{2}+b_{1}-1\right)}{\beta_{2}}, \\
& x_{19}=\frac{\left(\alpha_{1}-1\right) \alpha_{2}\left(\beta_{2}+\beta_{1}-1\right)}{\alpha_{1} \beta_{2}} \text {, } \\
& x_{20}=\left(\alpha_{1} \alpha_{2} \beta_{2}+\alpha_{2} \beta_{2}-3 \alpha_{1} \beta_{2}+\alpha_{1} \alpha_{2} \beta_{1}-2 \alpha_{1} \beta_{1}-\alpha_{1} \alpha_{2}-\alpha_{2}+2 \alpha_{1}\right) /\left(\alpha_{1} \beta_{2}\right) \text {, } \\
& x_{21}=\frac{\alpha_{2} \beta_{1}}{a_{1} \beta_{2}}, \quad x_{22}=1, \quad x_{23}=\frac{\left(\alpha_{2}-1\right)\left(\beta_{2}+\beta_{1}-1\right)}{\beta_{2}}, \\
& x_{24}=\frac{2\left(\alpha_{1}-1\right) \alpha_{2}\left(\beta_{2}+\beta_{1}-1\right)}{\alpha_{1} \beta_{2}}, \quad x_{25}=\frac{2\left(\alpha_{2}-\alpha_{1}\right)\left(\beta_{2}+\beta_{1}-1\right)}{\alpha_{1} \beta_{2}}, \\
& x_{26}=\frac{2\left(\alpha_{2}-1\right)\left(\beta_{2}+\beta_{1}-1\right)}{\beta_{2}}, \\
& x_{27}=\left(\alpha_{1} \alpha_{2} \beta_{2}-2 \alpha_{1} \beta_{2}+\alpha_{1} \alpha_{2} \beta_{1}-\alpha_{2} \beta_{1}-\alpha_{1} \beta_{1}-\alpha_{1} \alpha_{2}+\alpha_{1}\right) /\left(\alpha_{1} \beta_{2}\right) \text {, } \\
& x_{28}=\frac{\alpha_{2} \beta_{1}}{\alpha_{1} \beta_{2}}, \quad x_{29}=1, \quad x_{30}=\frac{2\left(\alpha_{2}-1\right)\left(\beta_{2}+\beta_{1}-1\right)}{\beta_{2}} \text {, }
\end{aligned}
$$




$$
\begin{array}{ll}
x_{31}=\frac{\left(\alpha_{1}-1\right) \alpha_{2}\left(\beta_{2}+\beta_{1}-1\right)}{\alpha_{1} \beta_{2}}, & x_{32}=\frac{\left(\alpha_{2}-\alpha_{1}\right)\left(\beta_{2}+\beta_{1}-1\right)}{\alpha_{1} \beta_{2}}, \\
x_{33}=\frac{3\left(\alpha_{2}-1\right)\left(\beta_{2}+\beta_{1}-1\right)}{\beta_{2}}, & x_{34}=0 .
\end{array}
$$

\section{Some closing challenges}

Here is a challenge that is motivated by recent work on matrix valued orthogonal polynomials. This subject was initiated by M.G. Krein a long time ago, see [29, 30]. There has been quite a bit of work in terms of producing explicit examples of these polynomials. For a representative sample see [3, 6, 7, 11, 15, 16, 17, 33, 34]. Most of this work deals with looking for examples that would enjoy the appropriate bispectral property. On the surface of it, there is little relation between these polynomials and the topics discussed here. But appearances can be deceiving. In a recent paper [12] I noticed how the example in [11] has a probabilistic interpretation (still waiting to be unravelled) and leads to nonnearest neighbours transitions on the nonnegative integers. This goes beyond the usual birth-and-death processes for which there is a well known relation to orthogonal polynomials, see [1, 2, 23, 24, 26, 27]. In the same paper [12] one finds examples arising in the theory of matrix valued orthogonal polynomials (in one variable) that give rise to Markov chains in two dimensional discrete space.

Another challenge would be to find the appropriate concrete algebraic geometry that lies behind the relation among the variables $\alpha_{1}, \alpha_{2}, \beta_{1}, \beta_{2}$ and $p_{1}, p_{2}, p_{3}, p_{4}$. In particular this could have some bearing on the situation discussed in [22] where the spectrum of $\mathcal{K}$ is degenerate, see $(5.15)$.

One challenge mentioned already a couple of times consists in writing down the general formula for a five-term recursion formula that is displayed here only for $N=5$.

\section{Acknowledgments}

I am very thankful to a couple of referees who read the paper with great care and pointed out typos as well as ways to improve the presentation. The author was supported in part by NSF Grant \# 0603901.

\section{References}

[1] Andrews G., Askey R., Roy R., Special functions, Encyclopedia of Mathematics and Its Applications, Cambridge University Press, 1999.

[2] Askey R., Wilson J., Some basic hypergeometric orthogonal polynomials that generalize Jacobi polynomials, Mem. Amer. Math. Soc. 54 (1985), no. 319.

[3] Castro M., Grünbaum F.A., The algebra of matrix valued differential operators associated to a given family of matrix valued orthogonal polynomials: five instructive examples, Int. Math. Res. Not. 2006 (2006). Article ID 47602, 33 pages.

[4] Cooper R., Hoare M., Rahman M., Stochastic processes and special functions: on the probabilistic origin of some positive kernels associated with classical orthogonal polynomials, J. Math. Anal. Appl. 61 (1977), 262-291.

[5] Duistermaat J.J., Grünbaum F.A., Differential equations in the spectral parameter, Comm. Math. Phys. 103 (1986), 177-240.

[6] Duran A.J., Grünbaum F.A., Orthogonal matrix polynomials satisfying second order differential equations, Int. Math. Res. Not. 2004 (2004), no. 10, 461-484.

[7] Duran A., van Assche W., Orthogonal matrix polynomials and higher order recurrence relations, Linear Algebra Appl. 219 (1995), 261-280, math.CA/9310220.

[8] Dunkl C., Xu Y., Orthogonal polynomials of several variables, Cambridge University Press, 2001. 
[9] Ehrenfest P., Eherenfest T., Über zwei bekannte Einwände gegen das Boltzmannsche H-Theorem, Physikalische Zeitschrift 8 (1907), 311-314.

[10] Feller W., An introduction to probability theory and its applications, Vol. 1, 3rd ed., Wiley, 1967.

[11] Grünbaum F.A., Matrix valued Jacobi polynomials, Bull. Sci. Math. 127 (2003), 207-214.

[12] Grünbaum F.A., Random walks and orthogonal polynomials: some challenges, math.PR/0703375.

[13] Grünbaum F.A., The bispectral problem: an overview, in Special Functions 2000: Current Perspective and Future Directions, Eds. J. Bustoz et al., 2001, 129-140.

[14] Grünbaum F.A., Some bispectral musings, in The Bispectral Problem (Montreal, 1997), CRM Proc. Lecture Notes, Vol. 14, Amer. Math. Soc., Providence, RI, 1998, 11-30.

[15] Grünbaum F.A., Pacharoni I., Tirao J.A., Matrix valued spherical functions associated to the complex projective plane, J. Funct. Anal. 188 (2002), 350-441, math.RT/0108042.

[16] Grünbaum F.A., Pacharoni I., Tirao J.A., Matrix valued orthogonal polynomials of the Jacobi type, Indag. Mathem. 14 (2003), 353-366.

[17] Grünbaum F.A., Pacharoni I., Tirao J.A., Matrix valued orthogonal polynomials of the Jacobi type: the role of group representation theory, Ann. Inst. Fourier (Grenoble) 55 (2005), 2051-2068.

[18] Harnard J., Kasman A. (Editors), The bispectral problem (Montreal, 1997), CRM Proc. Lecture Notes, Vol. 14, Amer. Math. Soc., Providence, RI, 1998.

[19] Hoare M., Rahman M., Distributive processes in discrete systems, Phys. A 97 (1979), 1-41.

[20] Hoare M., Rahman M., Cumultive Bernoulli trials and Krawtchouk processes, Stochastic Process. Appl. 16 (1983), 113-139.

[21] Hoare M., Rahman M., Cumultive hypergeometric processes: a statistical role for the ${ }_{n} F_{n-1}$ functions, J. Math. Anal. Appl. 135 (1988), 615-626.

[22] Hoare M.R., Rahman M., A probabilistic origin for a new class of bivariate polynomials, SIGMA 4 (2008), 089, 18 pages, arXiv:0812.3879.

[23] Ismail M.E.H., Classical and quantum orthogonal polynomials in one variable, Cambridge University Press, 2005.

[24] Ismail M.E.H., Masson D.R., Letessier J., Valent G., Birth and death processes and orthogonal polynomials, in Orthogonal Polynomials, Editor P. Nevai, NATO Advanced Science Institutes Series C: Mathematical and Physical Sciences, Vol. 294, Kluwer Acad. Publishers, Dordrecht, 1990, 229-255.

[25] Kac M., Random wak and the theory of Brownian motion, Amer. Math. Monthly 54 (1947), 369-391.

[26] Karlin S., McGregor J., The classification of birth and death processes, Trans. Amer. Math. Soc. 86 (1957), 366-400.

[27] Karlin S., McGregor J., The Hahn polynomials, formulas and an applications, Scripta Math. 26 (1961), $33-46$.

[28] Koornwinder T., Two variable analogues of the classical orthogonal polynomials, in Theory and Applic. of Special Functions, Editor R. Askey, Academic Press, 1975, 435-495.

[29] Krein M.G., Fundamental aspects of the representation theory of Hermitian operators with deficiency index (m,m), AMS Translations, Series 2, Vol. 97, Providence, Rhode Island, 1971, 75-143.

[30] Krein M.G., Infinite J-matrices and a matrix moment problem, Dokl. Akad. Nauk SSSR 69 (1949), no. 2, $125-128$.

[31] Macdonald I., Affine Hecke algebras and orthogonal polynomials, Cambridge University Press, 2003.

[32] Schrödinger E., Kohlrausch F., Das Ehrenfestche Model der H-Kurve, Phys. Zeit. 8 (1907), 311-314.

[33] Sinap A., van Assche W., Orthogonal matrix polynomials and applications, J. Comput. Appl. Math. 66 (1996), 27-52.

[34] Tirao J., The matrix valued hypergeometric equation, Proc. Natl. Acad. Sci. USA 100 (2003), no. 14, $8138-8141$. 